\title{
Role of the cerebellum in visuomotor coordination
}

\section{Delayed eye and arm initiation in patients with mild cerebellar ataxia}

\author{
S.H. Brown ${ }^{1}$, K.R. Kessler ${ }^{2}$, H. Hefter ${ }^{2}$, J.D. Cooke ${ }^{1}$, H.-J. Freund ${ }^{2}$ \\ ${ }^{1}$ Department of Physiology, University of Western Ontario, London, Ontario, Canada \\ ${ }^{2}$ Neurologische Klinik der Heinrich-Heine-Universität, Moorenstrasse 5, Düsseldorf 1, Germany
}

Received: 24 August 1992 / Accepted: 12 February 1993

\begin{abstract}
The initiation of coupled eye and arm movements was studied in six patients with mild cerebellar dysfunction and in six age-matched control subjects. The experimental paradigm consisted of 40 deg step-tracking elbow movements made under different feedback conditions. During tracking with the eyes only, saccadic latencies in patients were within normal limits. When patients were required to make coordinated eye and arm movements, however, eye movement onset was significantly delayed. In addition, removal of visual information about arm versus target position had a pronounced differential effect on movement latencies. When the target was extinguished for $3 \mathrm{~s}$ immediately following a step change in target position, both eye and arm onset times were further prolonged compared to movements made to continuously visible targets. When visual information concerning arm position was removed, onset times were reduced. Eye and arm latencies in control subjects were unaffected by changes in visual feedback. The results of this study clearly demonstrate that, in contrast to earlier reports of normal saccadic latencies associated with cerebellar dysfunction, initiation of both eye and arm movements is prolonged during coordinated visuomotor tracking thus supporting a coordinative role for the cerebellum during oculo-manual tracking tasks.
\end{abstract}

Key words: Eye-arm coordination - Cerebellum - Visual feedback - Human

\section{Introduction}

Accuracy of voluntary limb movements made to visual targets requires precise temporal and spatial coordination of both the oculomotor and limb motor systems. During discrete visuomotor tasks, the eyes normally

Correspondence to: S.H. Brown, Center for Human Motor Research, Department of Movement Science, University of Michigan, 401 Washtenaw Ave, Ann Arbor, MI 48109-2214, USA foveate on the target well before limb movement is completed. It has thus been suggested that retinal information is essential for endpoint accuracy by not only providing spatial information concerning exact target location, but also by monitoring the location of the moving limb relative to the target (Prablanc et al. 1979, 1986; Fleischer 1989).

Extra-retinal information concerning eye position and nonvisual information from the moving limb may also be involved in the ongoing control of oculomanual movements. Of particular interest is the role of limb afferent information in the coordination of eye and limb movements. The accuracy of slow pursuit eye movements is enhanced when tracking one's moving limb as compared to tracking either an external target (Steinbach and Held 1968; Mather and Lackner 1981) or one's arm following ischemic block (Gauthier and Hofferer 1976). More recently, Gauthier and colleagues have demonstrated a role for limb afferents in the coordination of eye and limb movements during slow smooth pursuit tracking in nonhuman primates (Gauthier and Mussa Ivaldi 1988). They suggested "the existence of a signal issued from kinaesthetic information of the arm which is used at higher control levels to coordinate the motion of the eyes with the hand" (p. 153).

The cerebellum is a likely candidate for such a coordination control centre. It is widely held that, for purposive limb movement, the cerebellum plays a major role, both in the timing of descending motor commands and in monitoring and updating the evolving movement (Brooks 1986). For example, patients with cerebellar lesions take longer to initiate purposive limb movements on the affected side (Holmes 1917), an observation confirmed following discrete cerebellar lesions in nonhuman primates (Meyer-Lohmann et al. 1977; Miller and Brooks 1982; Spidalieri et al. 1983). In addition to delayed movement onset, movement decomposition and loss of terminal accuracy also contribute to the wellknown clinical description of cerebellar impairment. Recently, it has been shown that in cerebellar patients with little or no involvement of the upper extremities, the time 
course of simple, visually guided arm movements is disturbed (Brown et al. 1990).

In the case of saccadic eye movements, one of the most prominent findings is saccadic dysmetria. This has been observed following experimentally induced lesions of the cerebellar cortex (Aschoff and Cohen 1971; Ritchie 1976), deep nuclei (Vilis and Hore 1981; Vilis et al. 1983) and in patients with cerebellar cortical degeneration (Baloh et al. 1975; Zee et al. 1976) or following surgery for removal of cerebellar tumours (Selhorst et al. 1976). In contrast, however, initiation of saccadic eye movements is not disturbed following cerebellar damage (Zee et al. 1976; Vilis and Hore 1981).

Despite the wealth of experimental data supporting a direct role for cerebellar involvement in determining the metrics of independently generated eye and limb movements, what role the cerebellum may play in the coordination of these two motor systems during oculomanual tasks has received little attention. Vercher and Gauthier (1988) have recently demonstrated temporal uncoupling of the ocular and limb motor systems following lesions of the dentate nucleus in baboons. This was taken as evidence that the dentate, serving as a coordination control centre, combines limb kinaesthetic information with signals governing eye movements so as to produce appropriately linked eye and limb movements.

The following experiments were therefore designed to examine the initiation of eye and arm movements in patients with mild cerebellar ataxia. Many of the coordination studies to date have focussed on pursuit tracking in which slow tracking movements of the limb are accom- panied by smooth pursuit eye movements. In order to examine the effects of cerebellar dysfunction on discrete, goal-directed movements, a simple, step-tracking task requiring saccadic eye movements was employed in the present study. It will be shown that during eye-arm tracking, initiation of both the saccadic and limb motor systems is delayed in patients with cerebellar damage. Moreover, this delay is dependent upon the nature of visual feedback available during the movement. Changes in eye and arm movement trajectories under different visual feedback conditions will be described in a forthcoming paper. Part of the findings reported here have been presented in abstract form (Brown et al. 1991).

\section{Materials and methods}

\section{Subjects}

Experiments were performed on six male patients with cerebellar disease aged $31-70$ years (mean age 51 years). Patients were selected on the basis of having predominantly cerebellar symptoms with mild involvement of the upper limbs. Visual acuity was unimpaired. Exclusion criteria included severe head and trunk tremor, major brainstem involvement or demyelinating disease. All patients were ambulatory and gave informed consent for the procedures involved. Despite the use of rigorous screening criteria, it should be stressed that, as with most clinical studies, it is not possible to conclude with absolute certainty that the deficits seen here arise solely from lesions restricted to the cerebellum. Thus, for example, the absence of any clinical brainstem signs in this patient group does not preclude possible brainstem involvement.

Clinical data are summarized in Table 1. Five patients (TR, EH,

Table 1. Clinical data for cerebellar patients

\begin{tabular}{|c|c|c|c|c|c|c|c|c|c|}
\hline & Age & Sex & $\begin{array}{l}\text { Length of } \\
\text { illness }\end{array}$ & Diagnosis & Arm & Trunk & $\begin{array}{l}\text { Head/ } \\
\text { arm }\end{array}$ & $\begin{array}{l}\text { Stance/ } \\
\text { gait }\end{array}$ & Dysarthria \\
\hline $\mathrm{EH}$ & 49 & M & 6 years & Degeneration & 2 & 1 & 2 & $3 / 2$ & 2 \\
\hline TR & 31 & M & 5 years & Degeneration & 2 & 1 & 2 & $3 / 3$ & 2 \\
\hline $\mathrm{HH}$ & 57 & $\mathbf{M}$ & 2.5 years & Degeneration & 1 & 0 & 1 & $3 / 2$ & 0 \\
\hline FT & 70 & $\mathrm{M}$ & 14 days & Infarction & 1 & 0 & 2 & $1 / 1$ & 0 \\
\hline
\end{tabular}

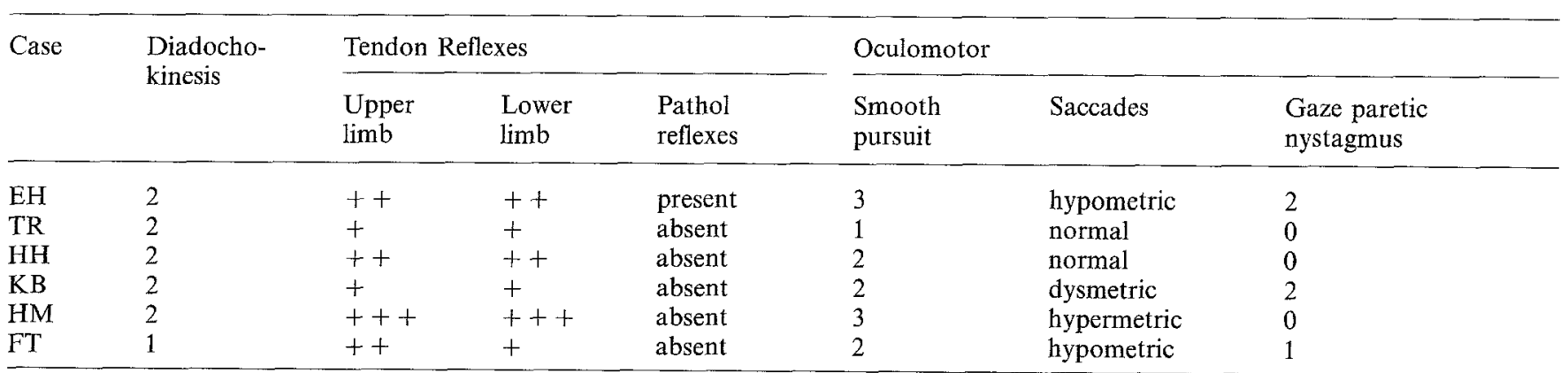

See text for detailed description of diagnosis

Rating of clinical symptoms: 0 , normal; $1-5$, mild to severe disturbance

Rating of tendon reflexes: 0 , no response; + , hypotonic; ++ , normal; +++ , clonus 
$\mathrm{HH}, \mathrm{KB}, \mathrm{HM}$ ) suffered from chronic cerebellar disease of $2.5-8$ years' duration. Following Harding's classification (Harding 1984), patients TR, HH, KB and HM were diagnosed as having idiopathic late-onset cerebellar ataxia. Patient FT presented acute cerebellar symptoms due to a stroke. MRI scans showed a left paramedian cerebellar infarct including parts of the vermis. In cases where symptoms were bilateral (TR, EH, HH, KB), the dominant arm and ipsilateral eye were tested. In patients HM and FT the left arm and eye were tested.Control experiments were performed on six subjects with no known history of motor dysfunction (five male, one female, age $30-60$ years, mean age 47 years).

\section{Experimental paradigm}

The experimental setup used in these studies was similar to that used previously (Brown et al. 1990). Subjects were seated comfortably and grasped a manipulandum handle. The shoulder was abducted to $90 \mathrm{deg}$ and supported along the entire length of the forearm. The manipulandum was pivoted beneath the elbow and moved freely in the horizontal plane. A contoured head and back rest was used to stabilise the head and trunk. Experiments were performed in a darkened room with the subject's arm blocked from view.

Target and handle position were displayed on a television screen placed $1 \mathrm{~m}$ in front of the subject. The target (displayed as two thin vertical lines $30 \mathrm{~mm}$ high and $15 \mathrm{~mm}$ apart) switched every $5 \mathrm{~s}$ between two fixed positions \pm 20 deg about an elbow angle of 90 deg. Position of the manipulandum handle (and thus of the subject's arm) was displayed as a thin vertical line. Target switch was accompanied simultaneously by a brief auditory tone. Accurate tracking of the display produced a 40 deg elbow movement and an eye movement of approximately $15 \mathrm{deg}$.

During each experimental session, tracking was performed with the eyes only and with both the eyes and arm. In the eyes only condition, subjects were instructed to visually track a step change in target position. In the eye-arm coordination task, subjects made movements under three different visual feedback conditions. This is shown schematically in Fig. 1. Under normal visual feedback conditions, both target and handle cursor remained visible throughout each movement trial. During handle blanking the handle cursor was extinguished (dashed line) for $3 \mathrm{~s}$ at the time of target switch. During target blanked trials the target cursor was extinguished for $3 \mathrm{~s}$ at the time of target switch. In both handle and target blanked conditions, the blanked cursor reappeared prior to the subsequent movement, so that subjects could correct for any positional errors. Within any given trial, the feedback condition remained constant. In handle and target blanked trials, blanking occurred randomly during $50 \%$ of both flexion and extension movements.

Under normal visual feedback conditions, subjects were instructed to place the handle cursor within the target zone by moving the handle quickly but accurately. Emphasis was placed on making one single, continuous movement to the target. During handle and target blanked trials, subjects were instructed to move the handle to the correct target position. To ensure that subjects understood the task, each subject made three to five movements under each feedback condition prior to data collection. Each trial consisted of ten flexion and ten extension movements. Two sets of trials were performed under normal visual feedback conditions, followed sequentially by three sets of handle blanked and four sets of target blanked trials. Each experimental session lasted $30-40 \mathrm{~min}$.

\section{Data recording and analysis}

Angular position and velocity of the manipulandum handle were recorded respectively from a potentiometer and tachometer mounted beneath the pivot point of the handle. Horizontal eye movements were recorded by DC electro-oculography (EOG) using $\mathrm{Ag}-\mathrm{AgCl}$ electrodes attached to the inner and outer canthi of the eye ipsilateral to the tested arm. The electrodes were allowed to stabilise 30 min prior to data recording. EOG signals were calibrated by having subjects fixate on targets on the screen 5,10 and $20 \mathrm{~cm}$ on either side of the midline of the tested eye. EOG calibration was done prior to each tracking condition and at the end of the experimental session.

All data were digitised with an effective sampling rate of $500 \mathrm{~Hz}$. The EOG signal was amplified and filtered online (DC $300 \mathrm{~Hz}$ ) prior to digitising. Individual eye movement onset times were deter-
Normal Visual Feedback

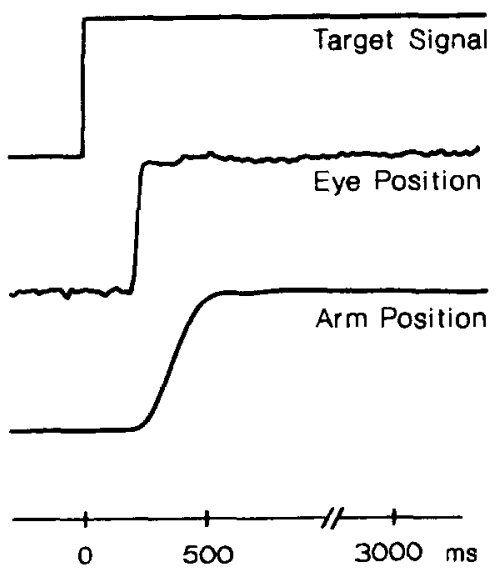

Fig. 1. Schematic diagram of feedback conditions used in eye-arm tracking tasks. In each set of records target signal, horizontal eye (EOG) position and manipulandum handle (arm) position are shown. Under normal visual feedback conditions, both target and handle signals remained visible throughout each movement trial. In handle blanked and target blanked conditions, upward arrows indi-
Handie Blanked
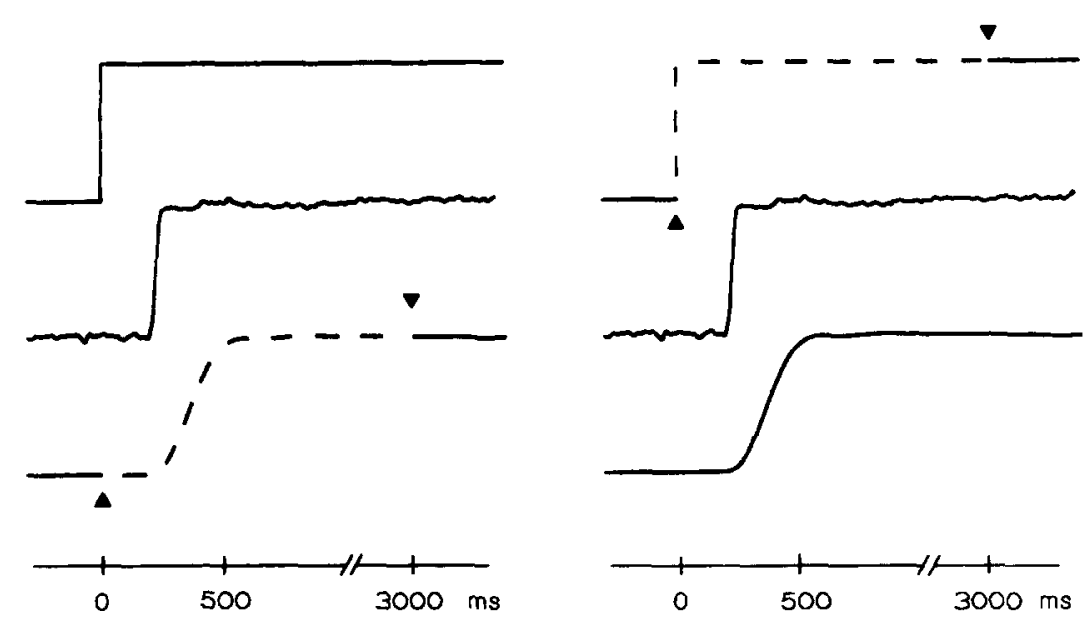

cate the time at which the signal was extinguished (dashed lines). In both handle and target blanked conditions, the blanked signal reappeared after $3 \mathrm{~s}$ (downward arrows). Blanking occurred randomly during both flexion and extension movements; schematics show flexion movements only 
mined using interactive computer software. Arm movement onset times and position values were obtained automatically from the differentiated velocity signal using an acceleration threshold of 120 $\mathrm{deg} / \mathrm{s}^{2}$. Computer-selected timing points were confirmed by visual inspection of the plotted records.

\section{Results}

Effects of coupled eye and arm movements on saccade latency

In order to determine if movements involving both the visual and limb motor systems affected saccade onset, latencies associated with movements of the eyes only were compared with latencies during coordinated eye and arm tracking. When tracking with the eyes only (Fig. $2, \mathrm{E})$, both control and patient groups produced saccades approximately $230-240 \mathrm{~ms}$ following a change in target position. No significant differences between control subjects and cerebellar patients were detected for either inwardly (equivalent to arm flexion) or outwardly (arm extension) directed saccades.

In control subjects, coupled eye and arm movements (Fig. 2, E/A) had no significant effect on saccade latency. In contrast, however, saccade onset times increased significantly in the cerebellar patients. Compared to control values, cerebellar latencies increased by approximately $18 \%(P<0.05)$ and $30 \%(P<0.025)$ for inwardly and outwardly directed saccades respectively. Within-group comparisons of mean cerebellar latencies between the eyes only and eye-arm tracking conditions revealed a $19 \%$ increase $(P<0.05)$ for inwardly directed saccades. Despite increased latencies of outwardly directed saccades, group differences were nonsignificant.

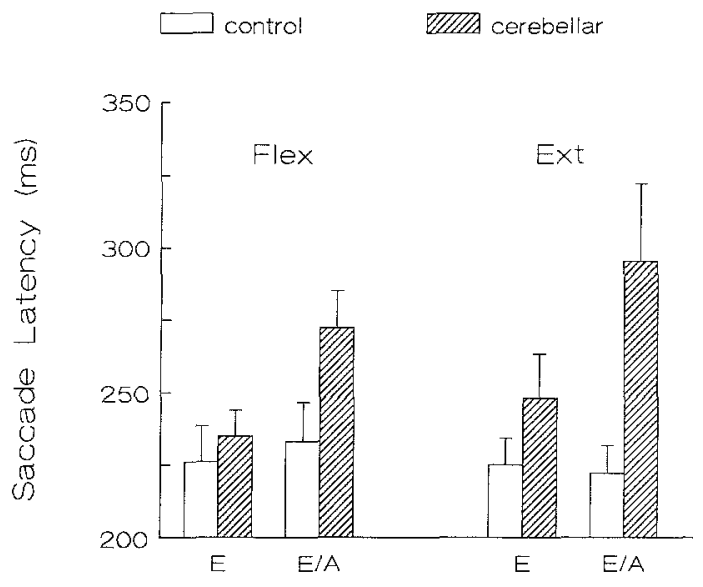

Fig. 2. Mean onset of horizontal saccades during eyes only $(E)$ and eye-arm $(E / A)$ tracking. Latencies are expressed relative to the time of target switch. Open and hatched bars represent the mean ( $\pm 1 \mathrm{SE})$ values for control $(n=6)$ subjects and cerebellar $(n=6)$ patients respectively. For arm flexion movements the corresponding eye movement was inwardly directed, and for extension movements, outwardly directed

Movement trajectories associated with different visual feedback conditions

Arm movements made by control subjects were characterised by smooth, continuous trajectories and were always accompanied by single, large amplitude saccades which, in general, preceded arm movement onset. This is shown in the upper sets of records in Fig. 3 where, for purposes of illustration, individual position traces have been synchronised around arm movement onset. The time course of both eye and arm movements made by control subjects was relatively unaffected following re-
Normal Visual Feedback
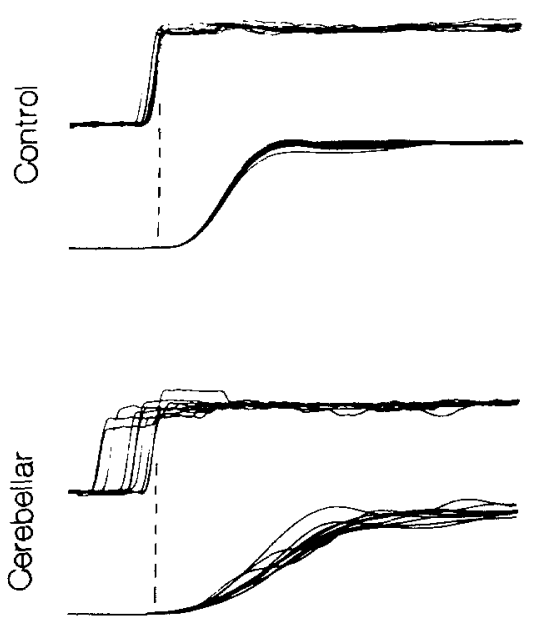

Fig. 3. Individual records of eye position associated with $40 \mathrm{deg}$ arm flexion movements made under different visual feedback conditions. Upper set of records was obtained from a control subject, the lower set from a cerebellar patient. In both cases, records of arm position
Handle Blanked
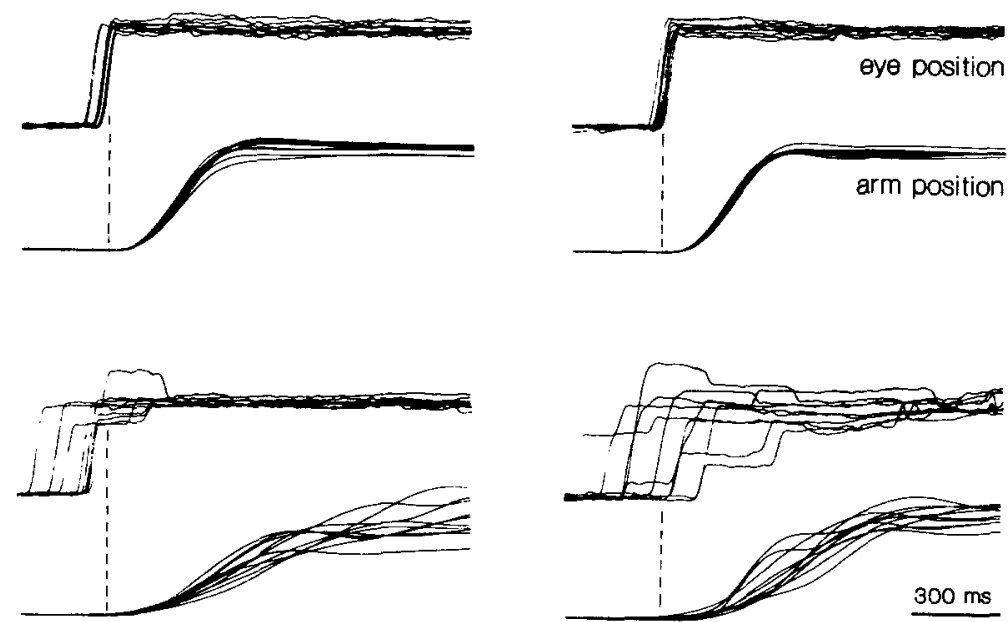

were synchronised around movement onset (dashed vertical lines). Eye position records were plotted relative to synchronised movement onset 

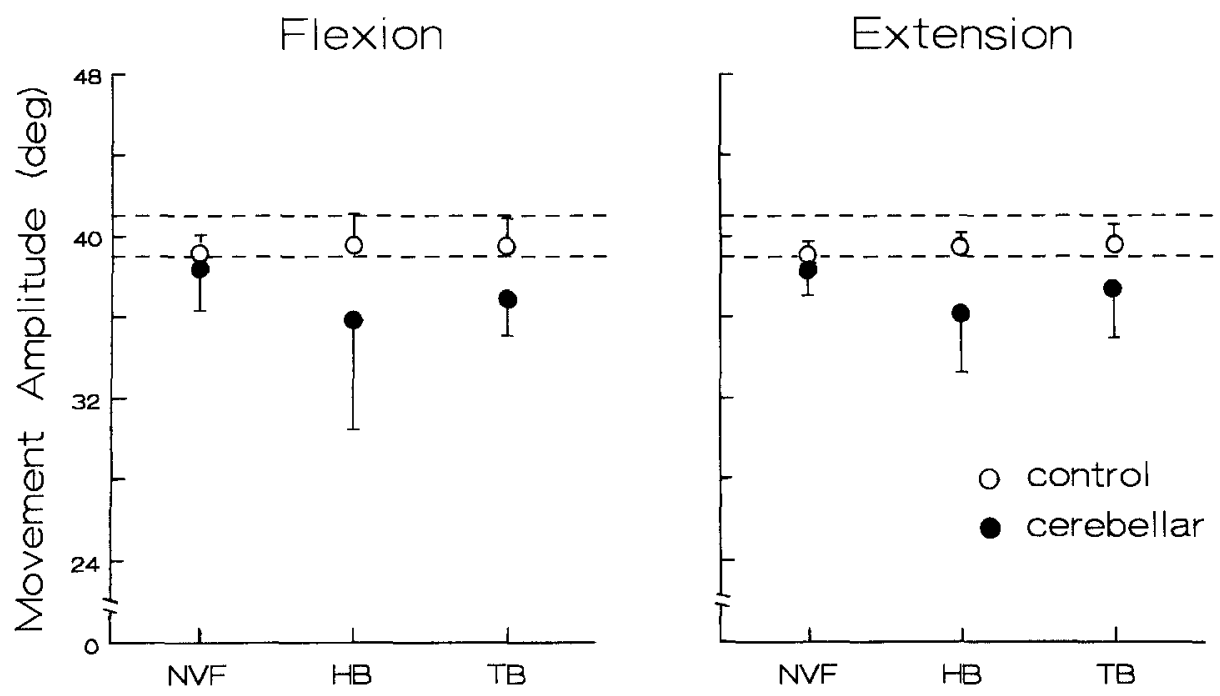

Fig. 4. Plots of arm movement amplitude under different visual feedback conditions. Open circles represent mean amplitude $( \pm 1 \mathrm{SE})$ for control subjects; filled circles, cerebellar patients. Dashed horizontal lines represent target zone (38-42 deg). NVF, normal visual feedback; $H B$, handle blanked; $T B$, target blanked

moval of either the handle or target cursor. In all cerebellar patients, however, marked alterations in both eye and arm movement trajectories were observed. Arm movements were often discontinuous, i.e. comprised two or more velocity peaks and total movement duration was almost twice that seen in control subjects. Qualitatively, arm movements were more irregular under both handle and target blanked conditions compared to movements made under normal visual feedback.

The corresponding eye movements were, in general, hypometric, comprising a large amplitude primary saccade and a smaller secondary saccade, particularly under normal visual feedback and handle blanked conditions. Dysmetria was most pronounced, however, when information regarding target location was removed. While most individual eye movements were multi-saccadic (i.e. comprising two or more hypometric saccades), patients occasionally produced a single, hypermetric saccade which was then followed by small amplitude saccades in the opposite direction. As is shown quantitatively in Fig. 9 , saccade onset times were more variable in the cerebellar group, particularly when target information was removed.

In control subjects, arm movement endpoint accuracy was unaffected following removal of either the handle or target cursor. Figure 4 shows mean movement amplitudes for control subjects and cerebellar patients for the different feedback conditions. Under normal visual feedback conditions, group differences were nonsignificant. In the patient group, removal of the handle or target cursor led to undershooting of the target in both movement directions. Absolute errors were small (approximately $10 \%$ of target amplitude) but statistically significant for handle blanked extension $(P<0.05)$ and target blanked flexion and extension $(P<0.05)$ movements.

\section{Initiation of eye and arm movements under different} feedback conditions

Mean onset of saccadic eye movements in control subjects was approximately $230 \mathrm{~ms}$ under normal visual feedback conditions. This is shown in Fig. 5 where all latency measurements have been determined relative to a change in target position. Although, on average, arm movement onset followed eye onset by approximately 15 $\mathrm{ms}$, the temporal ordering of eye and arm onset times was variable across individual control subjects. In three subjects eye onset preceded arm onset by approximately 50 $\mathrm{ms}$, in one subject eye and arm onset times were virtually identical and in two subjects arm movement preceded eye movement by approximately $20 \mathrm{~ms}$. Removal of the handle or target cursor had no significant effect on mean latencies of either the eye or the arm, regardless of the direction of the tracking movement. In addition, the temporal ordering of eye and arm movement onsets for each control subject was unaffected by different visual feedback conditions.

Compared to control values, both eye and arm onset times were significantly delayed in the cerebellar patients performing tracking movements under normal visual feedback conditions (eye, $P<0.02$; arm, $P<0.002$ ). Mean saccade onsets associated with both flexion (Fig. 5A) and extension (Fig. 5B) arm movements were 270 and $300 \mathrm{~ms}$ respectively. Onset of arm movement occurred approximately $65 \mathrm{~ms}$ later. Movement onset was further delayed in the cerebellar group when information concerning target location was removed. Compared to normal visual feedback values, saccade latency increased by approximately $20 \%(P<0.02)$ for movements made in either direction. Arm movement latencies also increased but mean differences were nonsignificant. Surprisingly, removal of handle (arm) position led to a small but significant decrease $(P<0.02)$ in eye onset times for movements made in either direction. Indeed, when compared to control values in the handle blanked condition, no significant group differences in saccade onset times were found. Mean arm onsets showed slight, but nonsignificant, decreases in the handle blanked condition.

This differential effect of removing target versus handle position was, as shown above, most dramatic for eye onset and was observed in all cerebellar patients for flexion arm movements and all but one patient for extension 

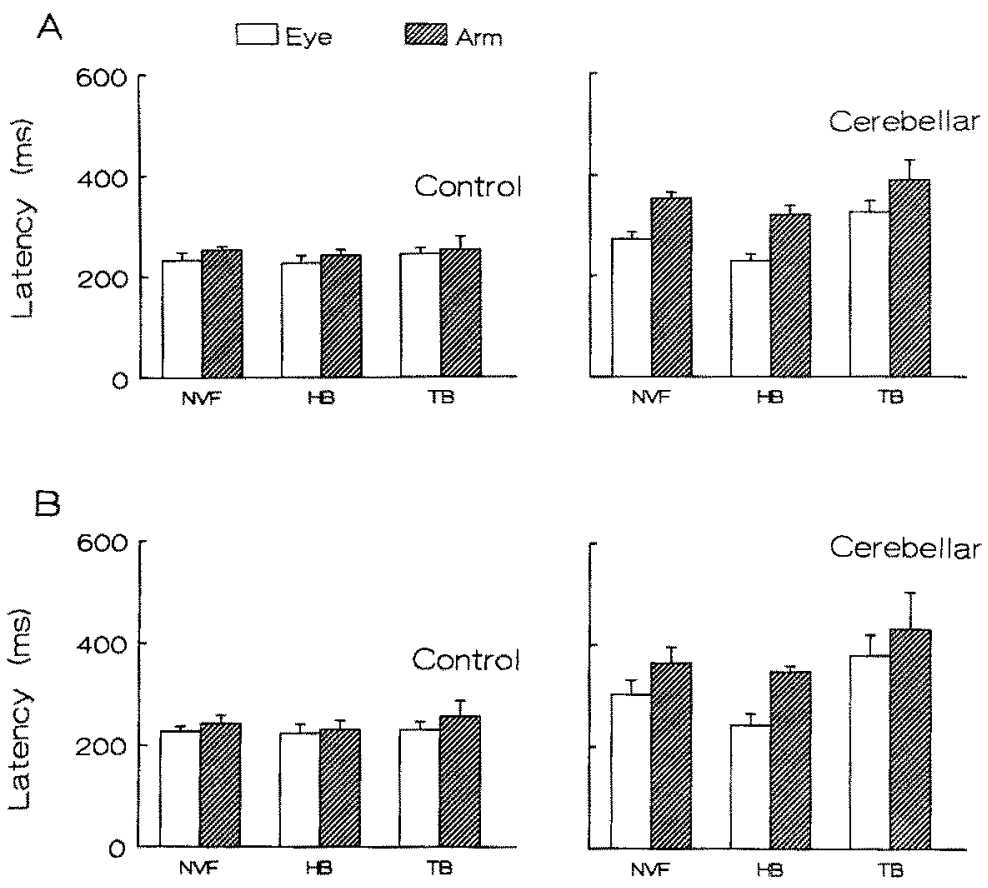

Fig. 5A,B. Mean onset times for movements made under different visual feedback conditions. In each histogram are shown mean ( $\pm 1 \mathrm{SE}$ ) latencies for eye (open bars) and arm (hatched bars) movements for control subjects (left-hand panels) and cerebellar patients (right-hand panels). A Data from flexion arm movements; $\mathbf{B}$ data from extension arm movements. All latency measurements were calculated relative to a switch in target position. Abbreviations as in Fig. 4
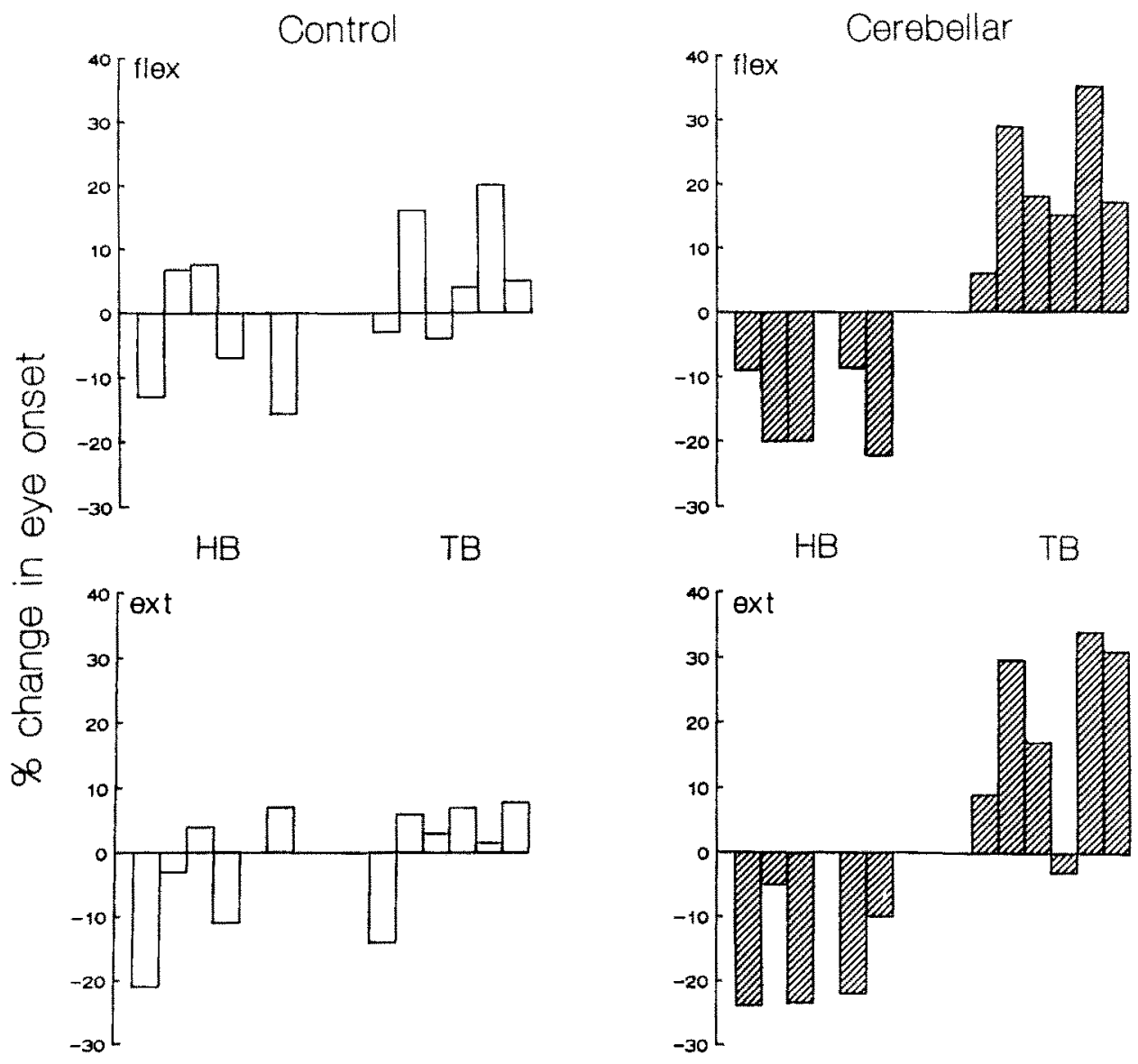

HB

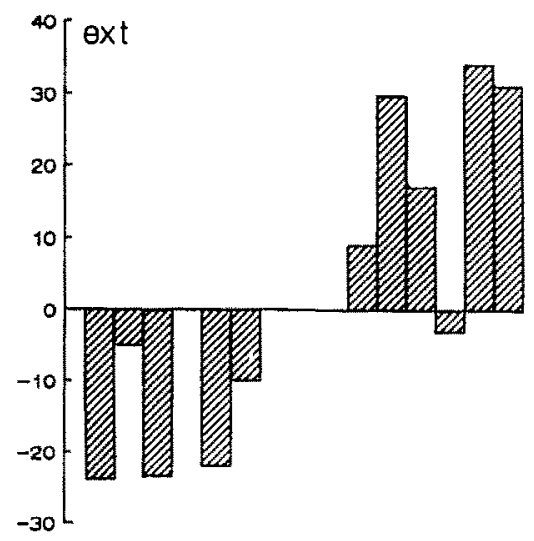

Fig. 6. Percentage change in eye movement onset during handle $(H B)$ and target $(T B)$ blanked conditions. Open bars in left-hand set of histograms represent individual control values; hatched bars (right-hand set) represent individual cerebellar patients. Positive values indicate an increase in onset times, negative values a decrease, relative to individual onset times associated with normal visual feedback conditions. Data from one control subject and one cerebellar patient were not vailable $f($. analysis in the $\mathrm{HB}$ condition. Each bar is the average of $15-20$ movements 


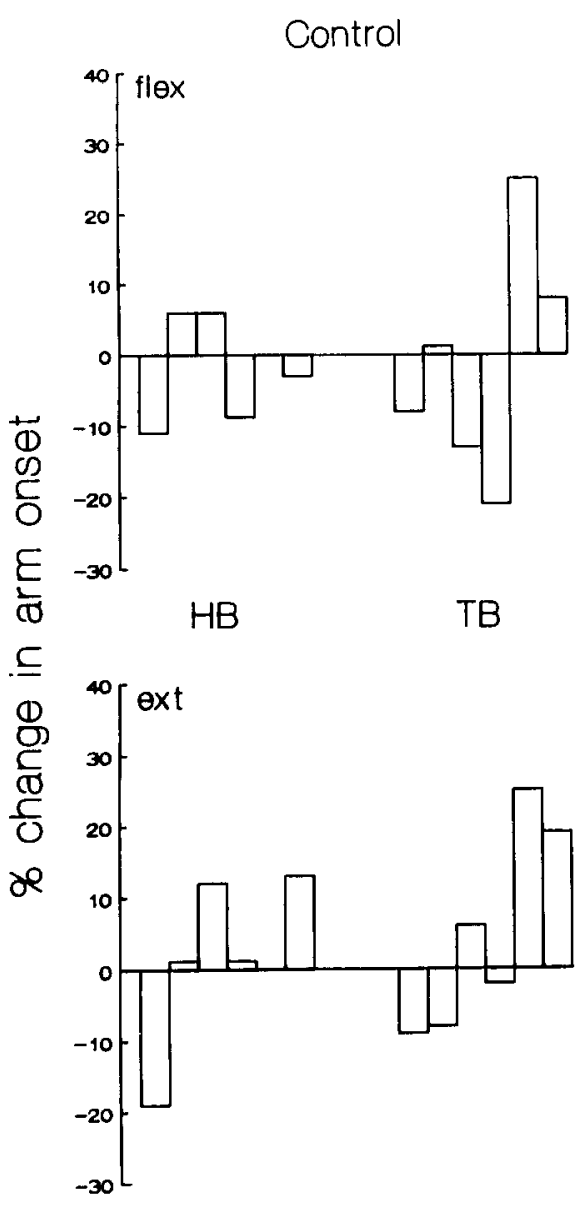

Cerebellar
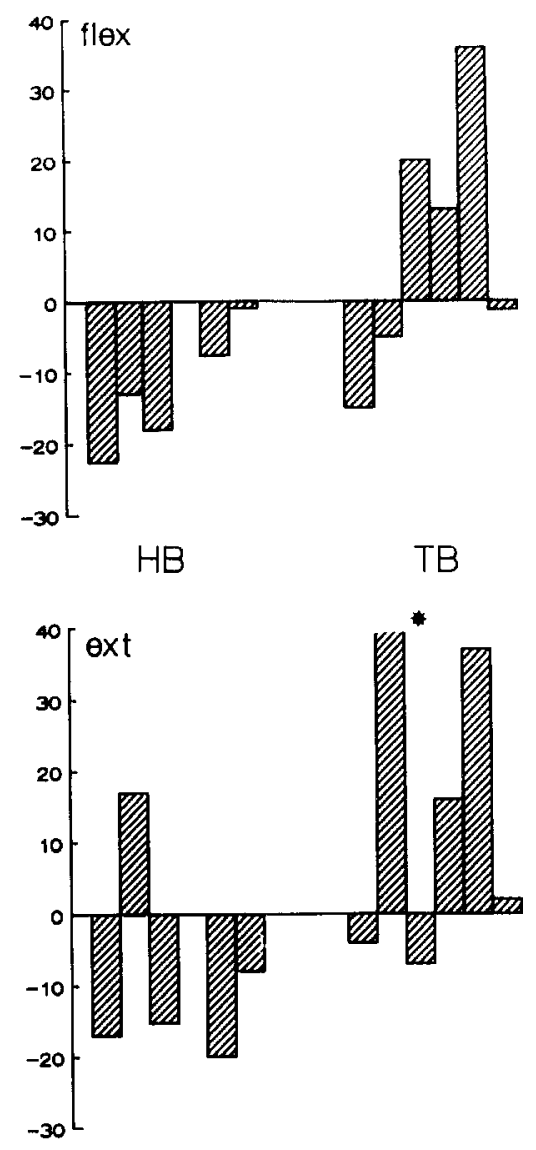

Fig. 7. Percentage change in arm movement onset during handle $(H B)$ and target $(T B)$ blanked conditions. Description of histograms as in Fig. 6. Bar marked by asterisk represents a $51 \%$ increase movements. Figure 6 shows the percentage change in eye latency from normal visual feedback values for individual control subjects and cerebellar patients. In all patients, eye movement onset decreased by $10-20 \%$ when visual information concerning handle (arm) position was removed. In contrast, removal of target location resulted in a $5-35 \%$ increase in eye onset times. In five of the six patients, increases in saccade latency occurred in both movement directions. In one patient (KB), no change was observed for outwardly directed eye movements; clinical examination, however, revealed no asymmetric disturbances in oculomotor function. In control subjects, changes in saccade latency were highly variable and inconsistent across feedback conditions and, as illustrated in Fig. 5, nonsignifcant.

In general, onset of arm flexion movements decreased in the cerebellar group when the handle cursor was removed. This is shown in Fig. 7. Only three patients, however, showed a corresponding increase with target blanking. Similar results were observed in extension movements. Compared to normal visual feedback values, mean arm onset times under handle and target blanked conditions were not statistically different. This was due to increased individual variability across both feedback conditions and movement directions. In general, however, the same pattern emerged for arm as for eye movement initiation in the cerebellar group: removal of visual target information led to an increase in movement onset while removal of handle (arm) position decreased onset times.

\section{Variability of eye and arm onset times}

For any given subject in either test group, saccade and arm onset times were poorly correlated when compared on a movement-to-movement basis. Distribution of individual latencies are shown for one representative control subject (A) and two cerebellar patients (B,C) in Fig. 8. Latencies for control subjects were tightly coupled and showed little variability under all visual feedback conditions. In contrast, cerebellar patients often exhibited latencies ranging over $200 \mathrm{~ms}$ for eye (Fig. 8B,C; NVF) and over $500 \mathrm{~ms}$ for arm (Fig. 8B,C; NVF,TB) movements. Despite such variability, eye and arm onset times for movements made under normal visual feedback conditions were poorly correlated, with correlation coefficients ranging from 0.06 to 0.56 . The one exception occurred in patient FT for extension movements (Fig. 8C; NVF). In this case, eye and arm onset times were linearly related $(r=0.88)$.

Eye and arm onset variability was greater in the cerebellar group compared to control subjects with variability increasing as movement onset times became more pro- 

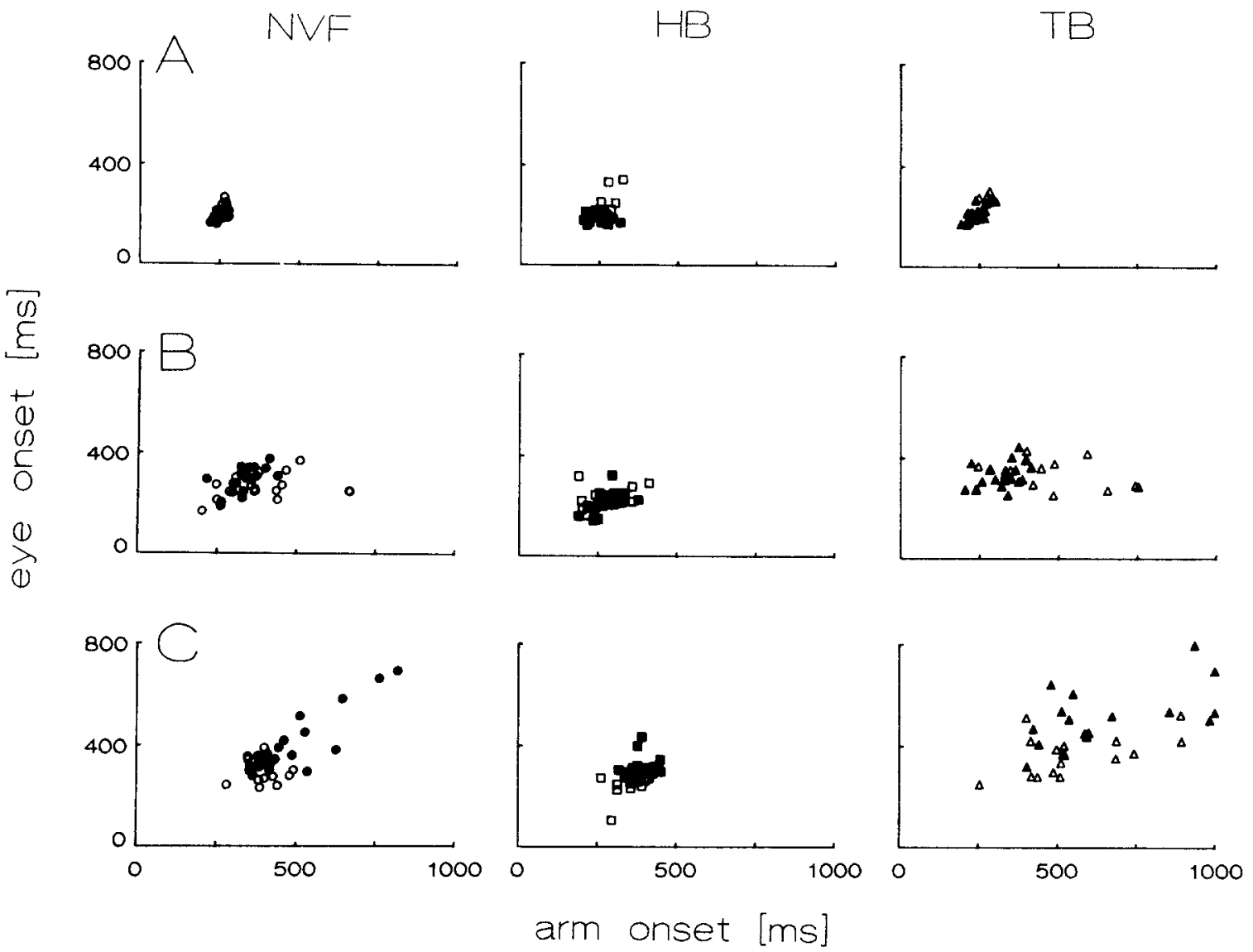

Fig. 8A-C. Correlation between eye and arm movement onset times under different visual feedback conditions. A Representative data from one control subject; B,C data from two cerebellar patients. Open symbols, data from individual flexion arm movements; filled circles, extension movements. Abbreviations as in Fig. 4
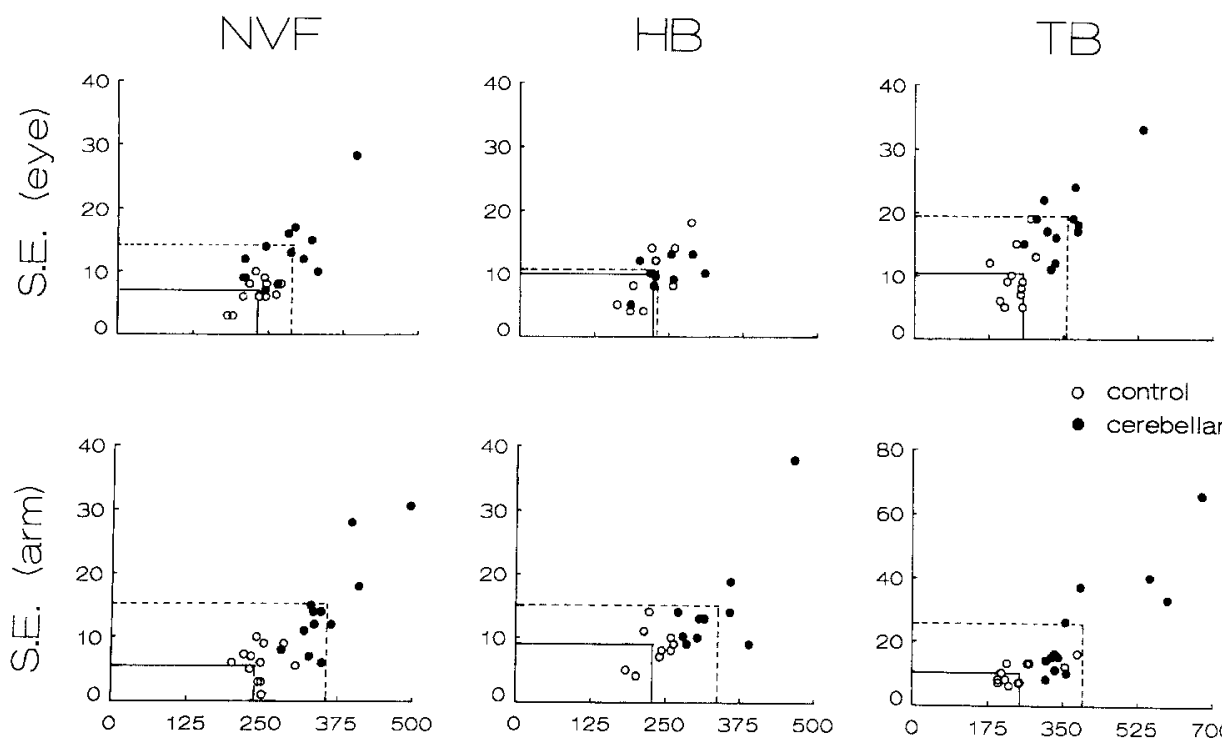

longed. This was most noticeable under normal visual feedback and target blanked conditions as shown in Fig. 9. When handle information was removed, however, little difference in the scatter of individual variability measures were observed for eye onset times between the two
Fig. 9. Movement onset variability as a function of eye (upper set of graphs) and arm (lower set) onset times (ms). Each data point represents the mean value obtained from individual control subjects (open circles) and cerebellar patients (filled circles). In each group, data from flexion and extension movements are included as separate data points but are not distinguishable, since no significant difference between flexion and extension variability was observed. Variability is expressed as one standard error (SE) of the mean. Mean scatter of data points is delineated by solid lines for control subjects and dashed lines for cerebellar patients. Note different vertical and horizontal scaling for righthand graphs (TB). Abbreviations as in Fig. 4 groups. This was due both to decreased variability in the patient group and increased variability in the control group. In contrast, variability in arm onset times was unaffected by the different feedback conditions. 


\section{Discussion}

Movement onset times associated with cerebellar dysfunction

Cerebellar participation in the generation of voluntary limb movements is well established. Since Holmes' early observations in man (Holmes 1917), lesions of the cerebellum are known to give rise to a variety of limb motor disturbances including deficient postural and force control, ataxia, tremor and a slowing of rapid alternating movements. Many of these signs are characterised by problems in movement timing. For instance, cerebellar patients often take longer to initiate a movement in response to a specific stimulus (Holmes 1917; Beppu et al. 1984; Inhoff et al. 1989), an observation confirmed by cerebellar lesion studies in nonhuman primates (MeyerLohmann et a1. 1977; Lamarre et al. 1978; Trouche and Beaubaton 1980; Chapman et al. 1986). In addition to delayed reaction times, it has been recently shown that patients with cerebellar lesions are unable to generate smooth, temporally symmetric movement profiles (Brown et al. 1990). Such kinematic irregularities may be related to an inability to correctly time descending motor commands governing activation of opposing muscle groups (Brown et al. 1989; Becker et al. 1990; Hallett et al. 1991; Hore et al. 1991).

In contrast, it is widely held that eye movement onset times are unaffected by cerebellar dysfunction. This has been demonstrated in patients with cerebellar dysfunction as well as in damage of cerebellar nuclei in nonhuman primates (Ritchie 1976; Selhorst et al. 1976; Zee et al. 1976; Vilis and Hore 1981, Vilis et al. 1983). Despite the presence of oculomotor deficits such as saccadic dysmetria and gaze nystagmus, saccadic reaction times during ocular tracking tasks are generally thought to be within normal limits. These observations have supported the view that initiation of saccadic eye movements does not involve cerebellar circuitry (Vilis and Hore 1984; Keller 1989).

The data obtained under normal visual feedback conditions in the present study also show that, when only the eyes are required to track a target, saccadic onset times are unaffected by cerebellar dysfunction. However, in contrast to control subjects, initiation of coupled eye and arm movements made by cerebellar patients are significantly prolonged. Thus, while the cerebellum may not be required for the generation of saccadic eye movements, it does play an important role in temporally coordinating the initiation of both the oculomotor and limb motor systems during visuomotor tasks.

As mentioned already, saccadic latencies in control subjects were unaffected when eye movements were accompanied by arm movements. This confirms earlier findings of Fischer and Rogal (1986) who observed no change in saccadic latencies when eye movements were coupled with hand movements. A study by Warabi et al. (1986) reported prolonged saccadic onset times in normal subjects during coupled eye and hand movements. In their paradigm, however, different target locations were used which appeared at irregular intervals. Thus, it is possible that spatiotemporal unpredictability in target presentation may have contributed to increased oculomanual latencies.

It should be noted that, in the present study, the temporal ordering of eye and arm movement onset times under normal visual feedback conditions varied considerably across control subjects, despite identical movement conditions and instructions. Whether such temporal variability represents individual differences in movement strategies or simply reflects a small sample size cannot be determined. In contrast to the common control hypothesis of oculomanual coordination (Herman et al. 1981; Biguer et al. 1982; Fisk and Goodale 1985), there is growing evidence supporting the view that saccadic and limb movements are generated independently, which could account for the individual differences in eye-arm latencies seen here (Gielen et al. 1984; Fischer and Rogal 1986; Carnahan and Marteniuk 1991).

\section{Coordinative role for the cerebellum during eye-limb tracking}

The present findings support the coordination model for oculomanual tracking recently put forth by Gauthier and colleagues (Gauthier et al. 1988). Based on observations that proprioceptive information from the moving limb improves accuracy of slow pursuit tracking movements (Gauthier and Hofferer 1976; Mather and Lackner 1981), they proposed that the cerebellum may serve as a coordination centre linking the oculomotor and limb motor systems during tasks requiring coordinated eye and limb movements. Coordination control would arise from the reciprocal exchange of information between the visual (retinal and extraretinal) and limb proprioceptive systems and would act "as a complement to the normal control in each subsystem" (Gauthier et al. 1988, p. 136). In this context, one would expect no change in saccadic latency when only the eyes are required to track the target - as observed in the present study - since the oculomotor system would be activated independently of the limb motor system and thus would bypass the coordination controller.

There is some evidence suggesting that, in addition to its role in movement planning and initiation, the lateral cerebellum may also contribute to eye movement production. Recently, Marple-Horvat and Stein (1990) recorded from neurons in the lateral cerebellar cortex of monkeys performing visually guided arm movements. The majority of neurons responded only to visual stimuli, or to eye or limb movements. However, some neurons were found which responded to both eye and arm movements, supporting a coordinative role for the cerebellum in visuomanual movements. Selective activation of dentate neurons in response to visual stimuli has been reported (Chapman et al. 1986), presumably involving projections from parieto-occipital areas of the cerebral cortex to lateral cerebellum via the brainstem (Glickstein et al. 1980). In addition, Vercher and Gauthier (1988) have shown that lesioning of the dentate nucleus in trained baboons abolishes eye-hand coordination during smooth pursuit tracking tasks. 
Although, in the present study, it was not possible to localise cerebellar damage, delayed onset times during eye-arm tracking support the view that the cerebellum is involved in coordinating the saccadic and limb motor systems. This particular aspect of motor timing, i.e. initiation of oculomanual movements, represents another example of the cerebellum as a central timekeeper. Thus, while different cerebellar regions may control timing functions which operate for specific motor systems over different time ranges (Keele and Ivry 1991), the findings reported here extend existing hypotheses concerning cerebellar involvement in the temporal organisation of voluntary movement (Braitenberg 1967; Kornhuber 1973; Ivry et al. 1988).

\section{Effects of visual feedback on eye and arm latencies}

Prolongation of movement onset times during visuomotor tracking was most noticeable when information concerning target location was removed (target blanked condition). This was particularly noticeable for eye movements where saccadic onset times increased by approximately $60 \mathrm{~ms}$ compared to movements made to continuously visible targets. This delay in initiation of memory guided saccades and associated arm movements suggests that, with cerebellar dysfunction, initiation of goal-directed, oculo-manual movements relies heavily on the presence of a visual target.

Various cortical and subcortical brain regions are thought to be involved in the integration and transformation of visual signals into internal spatiotemporal coordinates which are then used to generate appropriate oculomotor responses to remembered targets. Posterior parietal cortex, particularly the lateral intraparietal (LIP) area, contains neurons whose discharge is related to memory guided saccades (Gnadt and Anderson 1988). Latency and accuracy of memory guided saccades have been found to be impaired following lesioning of the frontal eye fields (Deng et al. 1986), as well as the substantia nigra and superior colliculus (Hikosaka and Wurtz 1989). Whether the present findings indicate an impairment in coding of visuospatial coordinates or simply reflect increased processing time in which stored coordinates are accessed by the cerebellum cannot be ascertained. Since, however, the cerebellum receives a variety of visually related inputs from such areas as posterior parietal cortex and the superior colliculus, it is possible that initiation of movements made to remembered targets represents delays in cerebellar integration of target-related, spatial information and temporal coordination of the oculomotor and limb motor systems.

Lastly, it is of interest to note that removal of handle (and thus arm) position appeared to actually facilitate eye movement onset in the cerebellar group. In this situation, information regarding limb position at the time of movement onset is provided primarily by proprioceptive afferents. It is thus possible that, by utilising proprioceptive in place of retinal information about static limb position, the processing time required to coordinate initiation of eye and limb movements to visual targets is reduced in patients with cerebellar dysfunction.

In summary, the results of the present study clearly demonstrate that, in contrast to earlier reports of ocular abnormalities associated with cerebellar dysfunction, onset of both saccadic and arm movements is prolonged during coordinated visuomotor tracking. These results are in agreement with other experimental findings supporting a coordinative role for the cerebellum during oculomanual tracking tasks.

Acknowledgements. The work was supported by the Deutsche Forschungsgemeinschaft (SFB 200, B3), and an Alexander Von Humboldt research fellowship (S.H. Brown).

\section{References}

Aschoff JC, Cohen B (1971) Changes in saccadic eye movements produced by cerebellarcortical lesions. Exp Neurol 32:123-133

Baloh RW, Konrad HR, Honrubia V (1985) Vestibuloocular function in patients with cerebellar atrophy. Neurology 25:160-168

Becker WJ, Kunesch E, Freund H-J (1990) Coordination of a multijoint movement in normal humans and in patients with cerebellar dysfunction. Can J Neurol Sci 17:264-274

Beppu H, Suda M, Tanaka R (1984) Analysis of cerebellar motor disorders by visually guided elbow trackingmovements. Brain 107:787-809

Biguer Jeannerod M, Prablanc C (1982) The coordination of eye, head and arm movements during reaching at a single visual target. Exp Brain Res 46:301-304

Braitenberg V (1967) Is the cerebellar cortex a biological clock in the millisecond range? Prog Brain Res $25: 334-346$

Brooks VB (1986) The neural basis of motor control. Oxford University Press, New York pp 256-289

Brown SH, Hefter H, Cooke JD, Freund H-J (1989) Duration of movement-related EMG activity in patients with mild cerebellar dysfunction. Soc Neurosci Abstr 15:473.2

Brown SH, Hefter H, Mertens M, Freund H-J (1990) Disturbances in human arm movement trajectory due to mild cerebellar dysfunction. J Neurol Neurosurg Psychiat 53:306-313

Brown SH, Kessler K, Hefter H, Cooke JD, Freund H-J (1991) Initiation of eye and arm movements in patients with mild cerebellar ataxia. IBRO Proc P65.14

Carnahan H, Marteniuk RG (1991) The temporal organization of hand, eye and head movements during reaching and pointing. $J$ Mot Behav 23:109-119

Chapman CE, Spidalieri G, Lamarre Y (1986) Activity of dentate neurons during arm movements triggered by visual, auditory, and somesthetic stimuli in the monkey. J Neurophysiol 55:203226

Deng S-Y, Goldberg ME, Segraves MA, Ungerleider LG, Mishkin M (1986) The effect of unilateral ablation of the frontal eye fields on saccadic performance in the monkey. In: Keller E, Zee DS (eds), Adaptive processes in the visual and oculomotor systems. Pergamon, Oxford, pp 201-208

Fischer B, Rogal L (1986) Eye-hand-coordination in man: a reaction time study. Biol Cybern 55:253-261

Fisk JD, Goodale MA (1985) The organization of eye and limb movements during unrestricted reaching to target in contralateral and ipsilateral visual space. Exp Brain Res 60:159-178

Fleischer AG (1989) Planning and execution of hand movements. Biol Cybern 60:311-321

Gauthier GM, Hofferer JM (1976) Eye tracking of self-moved targets in the absence of vision. Exp Brain Res 26:121-139

Gauthier GM, Mussa Ivaldi F (1988) Oculo-manual tracking of visual targets in monkey: role of the arm afferent information in 
the control of arm and eye movements. Exp Brain Res 73:138154

Gauthier GM, Vercher J-L, Mussa Ivaldi F, Marchetti E (1988) Oculo-manual tracking of visual targets: control learning, coordination control and coordination model. Exp Brain Res 73:127-137

Gielen CCAM, Van den Heuvel PJM, Van Ginsbergen JAM (1984) Coordination of fast eye and arm movements in a tracking task. Exp Brain Res 56:154-161

Glickstein M, Cohen JL, Dixon B, Gibson A, Hollins M, Labossiere E, Robinson F (1980) Corticopontine visual projections in macaque monkeys. J Comp Neurol 190:209-229

Gnadt JW, Anderson RA (1988) Memory related motor planning activity in posterior parietal cortex of macaque. Exp Brain Res $70: 216-220$

Hallett M, Berardelli A, Matheson J Rothwell J, Marsden CD (1991) Physiological analysis of simple rapid movements in patients with cerebellar deficits. J Neurol Neurosurg Psychiat 53:124-133

Harding AE (1984) The hereditary ataxias and related disorders Churchill Livingstone, Edinburgh, pp 166-173

Herman R, Herman R, Maulucci R (1981) Visually triggered eyearm movements in man. Exp Brain Res 42:392-398

Hikosada O, Wurtz RH (1989) The basal ganglia In: Wurtz RH, Goldberg ME (eds) Reviews of oculomotor research Vol. 3. The neurobiology of saccadic eye movements. Elsevier, Amsterdam, pp 257-282

Holmes G (1917) The symptoms of acute cerebellar injuries due to gunshot injuries. Brain 40:461-535

Hore J, Wild B, Diener H-C (1991) Cerebellar dysmetria at the elbow, wrist and fingers. J Neurophysiol 65:563-571

Inhoff AW, Diener HC, Rafal RD, Ivry R (1989) The role of cerebellar structures in the execution of serial movements. Brain 112:565-581

Ivry RB, Keele SW, Diener HC (1988) Dissociation of the lateral and medial cerebellum in movement timing and movement execution. Exp Brain Res 73:167-180

Keele SW, Ivry RB (1991) Does the cerebellum provide a common computation for diverse tasks? Ann NY Acad Sci 608: 179-211

Keller EL (1989) The Cerebellum. In: Wurtz RH, Goldberg ME (eds) Reviews of oculomotor research Vol. 3. The neurobiology of saccadic eye movements. Elsevier, Amsterdam, pp 391-411

Kornhuber HH (1973) Cerebellar control of eye movements. Adv Oto Rhino Laryngol 19:241-253

Lamarre Y, Bioulac B, Jacks B (1978) Involvement of the cerebellum in the initiation of fast ballistic movements in the monkey. Electroencephalogr Clin Neurophysiol 40:1038-1050

Marple-Horvat DE, Stein JF (1990) Neuronal activity in the lateral cerebellum of trained monkeys related to visual stimuli or to eye movements. J Physiol (Lond) 428:595-614
Mather JA, Lackner JR (1981) The influence of afferent, proprioceptive and timing factors on the accuracy of eye-hand tracking. Exp Brain Res 43:406-412

Meyer-Lohmann J, Hore J, Brooks VB (1977) Cerebellar participation in generation of prompt arm movements. J Neurophphysiol 40:1038-1050

Miller AD, Brooks VB (1982) Parallel pathways for movement initiation in monkeys. Exp Brain Res 45:328-332

Prablanc C, Echallier JF, Komilis E, Jeannerod M (1979) Optimal response of eye and hand motor systems in pointing at a visual target. I. Spatio-temporal characteristics of eye and hand movements and their relationships when varying the amount of visual information. Biol Cybern 35:113-124

Prablanc C, Pelisson D, Goodale MA (1986) Visual control of reaching movements without vision of the limb. I. Role of retinal feedback of target position in guiding the hand. Exp Brain Res 62:293-302

Ritchie L (1976) Effects of cerebellar lesions on saccadic eye movements. J. Neurophysiol 39:1246-1256

Selhorst JB, Stark L, Ochs AL, Hoyt WF (1976) Disorders in cerebellar motor control. I. Saccadic overshoot dysmetria: an oculographic, control system and clinico-anatomical analysis. Brain 99:497-508

Spidalieri G, Busby L, Lamarre Y (1983) Fast ballistic arm movements triggered by visual, auditory, and somesthetic stimuli in the monkey. II. Effects of unilateral dentate lesion on discharge of precentral cortical neurons and reaction time. J Neurophysiol 50:1359-1379

Steinbach MJ, Held R (1968) Eye tracking of observer-generated target movements. Science 161:187-188

Trouche E, Beaubaton D (1980) Initiation of a goal-directed movement in the monkey. Role of the cerebellar dentate nucleus. Exp Brain Res 40:311-321

Vercher J-L, Gauthier GM (1988) Cerebellar involvement in the coordination control of the oculo-manual tracking system: effects of cerebellar dentate nucleus lesion. Exp Brain Res 73:155166

Vilis T, Hore $\mathrm{J}$ (1981) Characteristics of saccadic dysmetria in monkeys during reversible lesions of medial cerebellar nuclei. J Neurophysiol 46:828-837

Vilis T, Hore J (1984) A comparison of disorders in saccades and in fast and accurate elbow flexions during cerebellar dysfunction. Prog Brain Res 64:207-215

Vilis T, Snow R, Hore J (1983) Cerebellar saccadic dysmetria is not equal in the two eyes. Exp Brain Res 51:343-350

Warabi T, Noda $\mathrm{H}$, Kato T (1986) Effect of aging on sensorimotor functions of eye and hand movements. Exp Neurol 92:686-697

Zee DS, Yee RD, Cogan DG, Robinson DA, Engel WK (1976) Oculor motor abnormalities in hereditary cerebellar ataxia. Brain 99:207-234 